this, fortunately, is already the case with the majority of clinies.

The Committee's recommendation for the educational provision of maladjusted children should be received with enthusiasm. It will not only save considerable money but will also be of greater service to maladjusted children than existing facilities. At present there are day special schools or classes specifically of maladjusted children in the areas of only three local education authorities. Over the country as a whole, little day provision has been made. Recent psychological findings indicate that there are obvious advantages in leaving a child in his own home during treatment. Since the restoration of harmony and understanding between parents and children must be one of the main purposes of treatment, it is most satisfactory to treat a child, if possible, while he remains in the natural environment of his own home. Treatment of him and of his parents can then go hand in hand; and there is no denger of a conflict being set up in his mind between the different standards of two environments. The co-operation of parents may be much easier to secure if their child can stay at home than if he is sent to a boarding establishment.

For maladjusted children local education authorities should make more use of day 'special' schools and part-time 'special' classes. No child should be sent to a boarding establishment if his education can be satisfactorily continued from home. The teachers should be allowed sufficient time for keeping in touch with the parents, the child guidance service and the ordinary schools from which the children come. All teachers of backward children will support the recommendation that staffing should be based on the principle that ordinarily a teacher cannot satisfactorily meet the needs of more than ten maladjusted children, and that for some purposes a group may need to be considerably smaller. No day or residential school should contain less than about twenty children nor more than about fifty. Strong support will be given to the recommendation that the Ministry of Education and local education authorities should constantly review the need for studying more maladjusted pupils over the age of eleven, "in particular boys with I.Q.'s below $85-90 \%$ and children suited to a grammar or a technical school course". Local education authorities should also consult with one another and other bodies to develop facilities for maladjusted children on a regional basis.

In the case of children whose activities lead to their appearance in juvenile courts, considerable care should be taken to see that the children are indeed maladjusted before being treated as such. A recent suggestion of the Magistrates' Association to the effect that meetings should be encouraged between magistrates, staffs of child guidance clinics and others connected with psychiatric work would be a valuable means of ensuring that court sentences would have greater prophylactic value. In general, too, support should be forthcoming for the Committee's recommendation that every child appearing before a juvenile court who is so handicapped as to need special educational treatment in a boarding school should be provided with residential treatment by the local education authority rather than be sent by the court to an approved school.

After outlining the size of the problem and practical measures needed to combat it, the Committee turns to the all-important but difficult task of developing a positive approach to mental health in the community as a whole. A number of its recommendations should be carried out forthwith. There should be, for example, close co-operation between child guidance clinics and child welfare centres. The function of health visitors should be modified, and they should be trained to advise parents on behaviour, nervous and other difficulties in children, as well as on matters of physical health. More clubs and discussion groups for parents should be established by schools, including nursery schools. Above all, the fundamental importance of the family as a whole should be borne in mind by those responsible for strengthening and developing the social services, and action designed to keep the family together should be regarded as one of the most important aspects of prevention.

The most valuable feature of this report is the way the Committee has made its way through the tangled mass of legislation and opinion which surrounds the problem of maladjusted children and has made recommendations which are practicable and attainable. Many of the recommendations will be very costly to carry out; but many of them will cost a lot more if they are not carried out. What is first needed is the building up of attitudes of mind required for the prevention and treatment of maladjustment. The five years taken to prepare this report will have been well spent if the thinking part of the community becomes familiar with its main arguments.

\section{PSYCHOSOMATIC RESEARCH AND BEHAVIOURAL SCIENCE}

T $\mathrm{T}$ is not often that one has the opportunity of reviewing two new journals and finding them of excellent quality within their respective fields. Both are quarterlies. The first, the Journal of Psychosomatic Research*, is to be devoted mainly to clinical studies if the present contents are an indication. These vary from such work as "Emotions and the Peripheral Vasomotor System" to "The Sedation Threshold as an Objective Index of Manifest Anxiety in Psychoneurosis". There are other papers on asthma and peptic ulcers. Such research should direct the atten. tion of the obstinately organically minded to the importance of mental states, and the psychologically orientated to the fact that the mind does not work in vacuo.

The scope of the other journal, Behavioral Science $\dagger$, is stated in the introductory editorial. The editors ask: "Can the scientific method solve the larger, more pervasive questions about man as well as the smaller, more particular ones? Is the tool with which man has won his victories over the physical world applicable to uncovering the laws which govern man's conduct, the deepest causes of our strife and our harmony? If fragments of multiple sciences were brought together in a unitary behavioral science and all the separate skills focused on the study of human behavior, perhaps the time required to find the answers to these questions could be reduced. The uniformities among disciplines could be recognised;

* Journal of Psychosomatic Research, 1, No. 1 (1956). Pp. $96+2$ plates. (London and New York : Pergamon Press, Ltd.) £5 (14 dollars) per year, or 708 . (9.80 dollars) for individual subscribers certifying that

the journal is for their private use. Md., and Ann Arbor, Mich.: Mental Health Research Institute, University of Michigan.) 6 dollars per year or 1.75 dollars per issue. 
better communication among them established; generalities of findings magnified; additional benefits derived from comparing theories in diverse fields, explaining similarities and differences, and the validity and applicability of empirical work increased by planning individual studies as components of an explicit mosaic of research strategy."

The result of this wide plan has been, perhaps inevitably, that the papers tend to be mainly mathematical. This may be valuable since there is a limit to clinical research, and without the aid of other sciences psychology and psychiatry will reach an impasse. The papers here suggest that there is little danger of this. They range from "Biological and Cultural Evolution" to a "Critique of Stochastic Models for Learning". This journal will be a joy to the mathematician and no doubt will draw many into the study of behaviour who would otherwise treat it as too vague to be of interest.

Clifford Aluen

\section{THE NEED FOR OBSERVATIONS IN THE OLDER BRANCHES OF ASTRONOMY}

F

OR his presidential address last year to the Royal Astronomical Society and since published (Mon. Not. Roy. Astro. Soc., 115, 2 ; 1955), Dr. J. Jackson took as his subject "The Need for Observations in the Older Branches of Astronomy, especially Double Stars", and discussed three branches with which he has been associated for about forty years: double stars, stellar parallax and meridian astronomy. After the First World War Dr. Jackson did a considerable amount of work on double stars, collecting and discussing the observations made during the preceding thirty years. The observing programme at that time was largely restricted to the Struve stars, but now that many new double stars have been catalogued and that there has been a great decrease in the number of observers, it is necessary, said Dr. Jackson, to review the position. Sufficient knowledge regarding the average masses and distances of ordinary stars is now available to decide on those stars that are worth observing at intervals of twenty years and to distinguish them from those that cannot be expected to reveal orbital motion of more than a degree or two in a century.

The importance of this is shown by the following considerations regarding the $\Sigma$ stars. These were all picked up during 1824-27 with a $9 \frac{1}{2}$-in. refractor from among the hundred and twenty thousand brightest stars north of Dec. $-15^{\circ}$, and while the original list contained more than three thousand double stars, after various adjustments and rejections there remained 2,659 brighter than about magnitude 9 and with a separation less than 32". Before these were put on the Greenwich observing list, most of them had been observed at several epochs, and when Dr. Jackson discussed them in 1922 he found as follows: relative motion was shown by 649 , undoubted orbital motion by 449 , mere optical doubles in 161 cases, and in 39 pairs showing rather large relative motion it was difficult to decide whether they formed binary or optical systems; it is suggested that while the majority probably belonged to the latter class, some may have been true binaries with a rather large parallax which could be confirmed by trigonometrical observations. A very important point is that 2,010 of these stars showed no certain relative motion of the components, and the great majority of these must be real binaries with periods of many thousand years, as Dr. Jackson shows by an analysis of the figures. His conclusion is that the 2,010 stars which had shown no relative motion by 1920 , although true binaries, scarcely need any further attention until the end of this century. The 449 showing orbital motion, and also the 39 for which the nature of the motion is uncertain, should, he thinks, be observed at intervals of ten or twenty years until an orbit can be computed or the pair proved merely optical.

Turning to the $\mathrm{O \Sigma}$ stars, which were discovered with the 15-in. refractor at Pulkovo mostly during 184.-42, Dr. Jackson said that, owing to the closeness of the components and relatively greater difference in brightness than those just considered, they are more difficult to see and observe accurately. Hussey re-observed them with the 12-in. and 36-in. refractors at Lick Observatory, and when Dr. Jackson discussed them in 1923 he found that 135 showed orbital motion, 15 were optical systems, 5 showed motion of uncertain nature, and 270 showed no motion. In spite of the fact that these stars had been observed over a shorter interval of time than the $\Sigma$ stars and, in addition, accurate observation was more difficult, yot they contained a greater percentage of stars of importance for the future, a fact which is attributable to the stars being closer and the components brighter. Dr. Jackson's final remarks on the subject were: "I therefore conclude that one of the most urgent needs of observational astronomy at the present time is the systematic re-observation of important double stars for which we have already detected the beginnings of orbital motion. The position may not be quite so bad as it appears as there may be a number of measures of double stars not yet published, and the absence of a general catalogue giving observations later than 1927 makes it difficult to estimate the true position. Nevertheless the reports of the International Astronomical Union do indicate a steadily decreasing number of measures". (After preparing his address, Dr. Jackson became aware of the measures of many close double stars made by Dr. Muller, of Strasbourg, during a four months visit to Lick Observatory, and a footnote to the published address directs attention to this, with a short account of the first orbits calculated for thirteen double stars.)

The position with regard to parallax determinations is very satisfactory when compared with that about half a century ago. In Newcomb's book "The Stars", published in 1904, there is a list of seventytwo stars for which parallaxes were known ; of these, there were fifteen about which it was noted that the results were subject to more doubt than usual, and one was stated to be entirely unreliable. In contrast to this, the Yale Observatory published in 1953 a "General Catalogue of Trigonometric Stellar Parallaxes", which gives the parallaxes of 5,822 stars from about ten thousand determinations; this is the outcome of the great international scheme, devised about forty years ago by Schlesinger, for the mass determination of parallaxes, and was taken up at a dozen observatories which had long-focus telescopes. Several of the telescopes which have completed their programmes are at present used for the intensive observations of the nearest stars in the search for companions which are too close or too faint to be 